\title{
The absorption and excitation spectroscopy of matrix-isolated atomic manganese: Sites of isolation in the solid rare gases
}

\author{
Martin A. Collier ${ }^{\mathrm{a})}$ and John G. McCaffrey ${ }^{\mathrm{b})}$ \\ Department of Chemistry, National University of Ireland-Maynooth, Maynooth, County Kildare, Ireland
}

(Received 13 September 2004; accepted 26 October 2004; published online 14 January 2005)

\begin{abstract}
This study collects information from absorption and luminescence excitation spectra recorded for $\mathrm{Mn}$ atoms isolated in the solid rare gases $\mathrm{Ar}, \mathrm{Kr}$, and $\mathrm{Xe}$ and presents an analysis of the site occupancy, based on the polarizabilities of the rare gases and the observed spectral shifts. Two thermally stable sites of isolation exist for atomic $\mathrm{Mn}$ in solid $\mathrm{Ar}$ and $\mathrm{Kr}$, while a single thermally stable site is present in $\mathrm{Mn} / \mathrm{Xe}$. Site occupancy assignments are based on the application of a polarizability model to the $z^{6} P_{5 / 2} \leftarrow a^{6} S_{5 / 2} ; z^{8} P_{5 / 2} \leftarrow a^{6} S_{5 / 2}$, and $y^{6} P_{5 / 2} \leftarrow a{ }^{6} S_{5 / 2}$ electronic transitions of atomic Mn. From an analysis of the observed RG matrix-to-gas phase energy shifts for $P \leftarrow S$ type transitions, this model allows the association of certain site types occupied by metal atoms in the rare gas solids. The required condition being a linear dependence of the matrix shifts with rare gas polarizability for those metal atoms "trapped" in a particular site type. Application of the polarizability model in conjunction with trends observed in site dominance, established a connection between the blue sites in $\mathrm{Ar}$ and $\mathrm{Kr}$ and the single site in Xe. Use of the known $\mathrm{Mg} \cdot \mathrm{RG}$ ground state bond lengths facilitated an identification of the sites of Mn atom isolation assuming the transference of the known $\mathrm{Mg} \cdot \mathrm{RG}$ bond lengths to the $\mathrm{Mn} \cdot \mathrm{RG}$ systems. Substitutional site occupancy of atomic $\mathrm{Mn}$ is assigned to the blue sites in $\mathrm{Ar}$ and $\mathrm{Kr}$ and the single site in $\mathrm{Xe}$, while tetra-vacancy site occupancy is assigned to the red sites in $\mathrm{Ar}$ and $\mathrm{Kr}$. Consistent with these assignments, Mn atoms in solid Ar show a preference for trapping in tetra-vacancy sites whereas in solid $\mathrm{Kr}$, single substitutional sites are preferred and in Xe, this is the only site observed. (C) 2005 American Institute of Physics. [DOI: 10.1063/1.1834568]
\end{abstract}

\section{INTRODUCTION}

The optical spectroscopy of matrix-isolated $n s^{2}$ metal atoms has lead to considerable insights into the behavior of ground and excited electronic state atoms in condensed matter. Our Group at Maynooth has reported on the luminescence spectroscopy of $\mathrm{Mg},{ }^{1} \mathrm{Zn},{ }^{2} \mathrm{Cd},{ }^{3}$ and $\mathrm{Hg}^{4}$ atoms isolated in solid rare gases and presented a localized $\mathrm{M} \cdot \mathrm{RG}_{18}(\mathrm{RG}$ $=\mathrm{Ar}, \mathrm{Kr}$, and $\mathrm{Xe}$ ) cluster model ${ }^{5}$ to probe ground and excited state interactions of the guest metal atom within the solid. This analysis allowed identification of site occupancy and simulation of the $\mathrm{M}-\mathrm{RG}$ interactions leading to the observed solid-state luminescence. The cluster model provides the link between the solid state luminescence and the behavior of the corresponding diatomic $\mathrm{M} \cdot \mathrm{RG}$ van der Waals complexes ${ }^{6,7}$ stabilized in cold supersonic expansions, thereby providing insight into the behavior of metal atoms in the condensed phase. In the past decade, the luminescence spectroscopy of atomic mercury has been studied in detail by Crepin and Tramer $^{8}$ at Orsay. Recently, the nonadiabatic dynamics of excited ${ }^{3} P_{1}$ state atomic mercury has been investigated by Chergui and co-workers ${ }^{9}$ using a hybrid quantal/classical dynamics calculation.

In this contribution the absorption and excitation spectroscopies of matrix-isolated atomic manganese are presented to extend the work done on the Group 2 and 12 metal atoms into the transition elements. This experimental work

\footnotetext{
${ }^{a)}$ Present address: Lab. Francis Perrin, CEA Saclay, France.

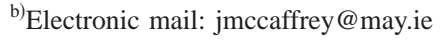

was motivated by the similarities between atomic manganese, which also exhibits an $n s^{2}$ ground electronic configuration and the M/RG systems $\left[\mathrm{M}=\mathrm{Mg},{ }^{1} \mathrm{Zn},{ }^{2} \mathrm{Cd}^{3}{ }^{3}\right.$ and $\left.\mathrm{Hg}^{4,6}\right]$ where the solid-state spectroscopy has been studied in detail. The lowest energy electronic configuration of atomic $\mathrm{Mn}$ is [Ar] $3 d^{5} 4 s^{2}$, giving rise to the spherically symmetric $a^{6} S_{5 / 2}$ ground state. The electronic states observed in absorption correspond to the "singletlike" $\left[3 d^{5} 4 s 4 p\left({ }^{1} P^{\circ}\right)\right] y^{6} P$ and "tripletlike" $\left[3 d^{5} 4 s 4 p\left({ }^{3} P^{\circ}\right)\right] z^{6} P$ states which occur at $279.91 \mathrm{~nm}\left(35725.85 \mathrm{~cm}^{-1}\right)$ and $403.42 \mathrm{~nm}(24788.05$ $\mathrm{cm}^{-1}$ ), respectively, in the gas phase. ${ }^{10,11} \mathrm{UV} / \mathrm{Vis}$ absorption spectra presented for each of the $\mathrm{Mn} / \mathrm{RG}(\mathrm{RG}=\mathrm{Ar}, \mathrm{Kr}$, and $\mathrm{Xe}$ ) systems indicated the existence of multiple trapping sites for the guest atom. Excitation spectra in particular allowed the extraction of photophysical properties of the trapping sites present, but not resolved in absorption spectra.

Manganese ${ }^{12}$ along with mercury, ${ }^{13}$ was one of the first metal atom systems to be investigated with the matrixisolation technique. Since the first report of the spectroscopy of $\mathrm{Mn} / \mathrm{RG}$ solids by Schnepp, ${ }^{12}$ Lee and Gutmacher, ${ }^{14}$ and Mann and Broida ${ }^{15}$ completed further work focusing on the UV/Vis atomic absorption spectroscopy in solid argon matrices deposited at $4.2 \mathrm{~K}$. A summary of these earlier reports on the absorption spectroscopy of $\mathrm{Mn} / \mathrm{RG}$ solids is given in Gruens' review. ${ }^{16} \mathrm{Mn} / \mathrm{RG}$ solids containing isolated Mn atoms and higher aggregates have been investigated by Vala and co-workers ${ }^{17}$ using the Magnetic Circular Dichroism (MCD) technique in Ar, Kr, and Xe. Many reports of spectroscopic analysis of the van der Waals, antiferromagnetic 
molecule manganese dimer ${ }^{18-20} \mathrm{Mn}_{2}$ and bimetallic clusters (containing $\mathrm{Mn}$ ) isolated in RG solids have appeared in the literature. ${ }^{21}$ In contrast, no reports of the luminescence spectroscopy of atomic manganese have appeared to date. However, one is given in an article ${ }^{22}$ to appear shortly.

The Mn/RG samples prepared during the course of this work are more "atomic" than those reported in previous studies. ${ }^{12-15,17-21}$ This is attributed to the increased control of the $\mathrm{Mn}$ vaporisation afforded by localized heating of $\mathrm{Mn}$ occurring with electron bombardment, compared with the bulk heating arising from resistive heating of $\mathrm{Mn}$ in Knudsen cells. The difficulties inherent in the vaporization of $\mathrm{Mn}$ and the controlled production of Mn/RG samples that have hampered previous attempts at assigning Mn clusters have been mentioned in some of the earlier reports. ${ }^{21}$ Concentration studies conducted in the present work, allowed distinction of atomic Mn absorption bands from Mn cluster species. The absorption spectra recorded allowed the identification of multiple thermally stable sites of isolation for $\mathrm{Mn}$ atoms isolated in solid $\mathrm{Ar}$ and $\mathrm{Kr}$ and a single site of isolation in $\mathrm{Xe}$. Excitation spectroscopy has been used extensively in the present study as it provides a much more discriminating method for site identification than absorption spectroscopy. As will be shown in the Mn/Ar system, resolution of overlapped absorption bands into individual site components is easily achieved by recording excitation spectra of site specific emission features.

In the following sections, the results of excitation spectroscopy recorded in the vicinities of the $y^{6} P_{5 / 2} \leftarrow a^{6} S_{5 / 2}$, $z^{6} P_{5 / 2} \leftarrow a^{6} S_{5 / 2}$, and $z^{8} P_{5 / 2} \leftarrow a^{6} S_{5 / 2}$ transitions of atomic manganese are presented for the first time. The polarizability model of Laursen and Cartland ${ }^{23}$ (L\&C) is applied to the matrix shifts obtained for these three transitions which allows association of bands arising from a given site occupancy. In addition, trends in the photophysical characteristics of the excitation bands observed for the $P \leftarrow S$ type transitions are discussed in relation to the site occupancy. The role of the "singlet" versus "triplet" nature of the excited states reached in the optical transitions are discussed with respect to the solid state interactions leading to the observed matrix shifts.

\section{RESULTS}

\section{A. UV/Vis absorption and excitation spectroscopy}

The apparatus used in the preparation of Mn/RG samples has been described ${ }^{2}$ in detail elsewhere. Specifics of the spectroscopic setup have also been presented ${ }^{4}$ recently. Except where specified, all the excitation spectra presented were recorded with dispersed, cw-lamp radiation. Thus, deuterium (Hamamatsu L6310 and a Cathodeon C713 power supply) and tungsten lamps (General Electric $30 \mathrm{~W}$, Model DZA) were used as the light sources to record both absorption and excitation spectra in the ultraviolet (UV, 180-400 $\mathrm{nm})$ and UV/Vis (350-600 $\mathrm{nm}$ ) spectral regions, respectively. A scanning $0.30 \mathrm{~m}$ monochromator (Acton Research Corporation, SpectraPro-300i) fitted with a ruled, 1200 grooves $/ \mathrm{mm}$ grating (blazed at $300 \mathrm{~nm}$ ) was used for wavelength selection. Pulsed laser radiation in the 490-535 nm

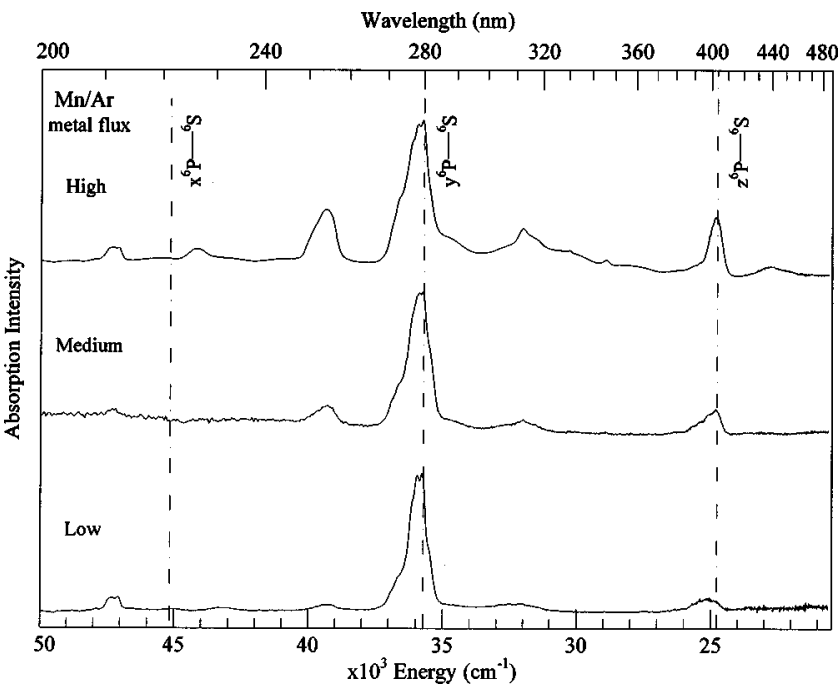

FIG. 1. UV/Vis absorption spectra recorded at $12 \mathrm{~K}$ for $\mathrm{Mn} / \mathrm{Ar}$ samples following deposition at $12 \mathrm{~K}$. The three spectra shown indicate the changes in the relative intensities of the observed bands with increased metal flux.

range was used to record excitation spectra of the spin forbidden $z^{8} P \leftarrow z{ }^{6} S \mathrm{Mn}$ atom transition. For this excitation the direct output of the oscillator in the Quantel TDL-90 dye laser was employed using Coumarin 500 as the dye material. The dye laser was pumped with the third harmonic $(355 \mathrm{~nm})$ of a Nd:YAG (Quantel YG-980E) laser operating at $10 \mathrm{~Hz}$. Excitation spectra were recorded with laser energy densities of $2.0 \mu \mathrm{J} / \mathrm{mm}^{2}$ by scanning the dye laser wavelength while monitoring a given emission band maximum. Ten laser shots were averaged for every wavelength position in the dye scan. The linewidth of the TDL-90 dye laser is $0.8 \mathrm{~cm}^{-1}$ at 560 nm.

Manganese vapor was generated by electron bombardment of the bulk metal using an ultrahigh vacuum Omicron (Model EFM3) evaporator. The electron beam was focused on irregular manganese chunks (Goodfellow, Johnson Matthey; purity $>99.5 \%$ ) with an average diameter of $3 \mathrm{~mm}$, contained in a $5 \mathrm{~mm}$ internal diameter molybdenum crucible. Under low metal loading conditions, the isolation of manganese atoms is favored. The absorption features of atomic manganese are assigned from their proximity to the gas phase positions, as the weak van der Waals interaction between the Mn atom and its immediate matrix environment results in only a slight deviation of the transition energy from that observed for the free atom. The assignment of atomic $\mathrm{Mn}$ absorptions is simplified by the presence of resolved threefold splitting pattern indicative ${ }^{24}$ of the dynamic JahnTeller effect. This effect has been observed for several matrix-isolated metal atoms including the ${ }^{3} P_{1} \leftarrow{ }^{1} S_{0}$ absorption of $\mathrm{Hg}$ atoms ${ }^{4}$ in solid $\mathrm{Xe}$.

\section{B. $\mathrm{Mn} / \mathrm{Ar}$}

Absorption spectra recorded for samples prepared by codepositing manganese vapor with argon on $\mathrm{CaF}_{2}$ window at $12 \mathrm{~K}$ are presented in Fig. 1. The three traces show the effect of increasing the metal flux, thereby providing a concentration study for the isolation of $\mathrm{Mn}$ in solid Ar. It was 
found that Ar was the most difficult of the solid rare gas hosts to achieve atomic isolation, but as shown in the bottom trace of Fig. 1, nearly exclusive atomic isolation was also achieved in this solid. The gas phase positions ${ }^{10}$ of the $x^{6} P_{5 / 2}, y^{6} P_{5 / 2}$, and $z^{6} P_{5 / 2} \leftarrow{ }^{6} S_{5 / 2}$ transitions are shown by the dashed vertical lines in Fig. 1. The dominant absorption feature observed for $\mathrm{Mn} / \mathrm{Ar}$ at $278.1 \mathrm{~nm}\left(35958 \mathrm{~cm}^{-1}\right)$ is assigned to the $y^{6} P_{5 / 2} \leftarrow{ }^{6} S_{5 / 2}$ transition of atomic Mn. This absorption feature is shifted by $232 \mathrm{~cm}^{-1}$ to higher energy from the gas phase (G.P.) position ${ }^{10}$ and exhibits a resolved threefold splitting pattern as well as high and low-energy shoulders at 273.0 and $281.5 \mathrm{~nm}$, respectively.

The absorption feature centered at $397.4 \mathrm{~nm}$ in the most dilute Ar sample (bottom trace) is assigned to the $z^{6} P_{5 / 2}$ $\leftarrow a^{6} S_{5 / 2}$ transition, based on its proximity to the location of this transition in the gas phase ${ }^{10}$ at $403.42 \mathrm{~nm}$. Inspection of the three traces in Fig. 1 reveals this absorption band exhibits a progressive shift to lower energy with increased metal loading. This redshift is ascribed to the production of $\mathrm{Mn}_{2}$ whose absorption at $402.3 \mathrm{~nm}$ coincidentally overlaps that of the atomic $z^{6} P_{5 / 2} \leftarrow a^{6} S_{5 / 2}$ line at $403.4 \mathrm{~nm}$ in the gas phase. The atomic Mn $z^{6} P_{5 / 2} \leftarrow a{ }^{6} S_{5 / 2}$ transition in $\mathrm{Ar}$ at $397.4 \mathrm{~nm}$ is blueshifted by $375 \mathrm{~cm}^{-1}$ from its G.P. position.

In earlier work the interpretation of the optical absorption spectroscopy of manganese atoms isolated in solid rare gases (especially Ar) has proven difficult, due to the large variety of species present on deposition in even the most dilute samples. In the literature, the $226.4 \mathrm{~nm}$ absorption feature has been assigned to the atomic $x^{6} P_{5 / 2} \leftarrow a^{6} S_{5 / 2}$ transition, due to the persistence of this band in the least concentrated samples prepared by Ozin and co-workers. ${ }^{21}$ From the concentration study shown in Fig. 1, the atomic $x^{6} P_{5 / 2}$ $\leftarrow a{ }^{6} S_{5 / 2}$ transition is identified at $212 \mathrm{~nm}$ (lower trace) and the $226 \mathrm{~nm}$ band (upper trace) is assigned to $\mathrm{Mn}_{2}$. With this assignment the atomic $x^{6} P_{5 / 2} \leftarrow a^{6} S_{5 / 2}$ transition is blue shifted by $2014 \mathrm{~cm}^{-1}$ from the gas phase position at $45156.11 \mathrm{~cm}^{-1}(221.45 \mathrm{~nm})$. The large shift on this transition can be rationalised in terms of the changes in the orbital occupancy accompanying it. Thus the excited $x^{6} P$ state arises from the $[\mathrm{Ar}] 3 d^{6}\left({ }^{5} D\right) 4 p$ electronic configuration, hence the $x^{6} P_{5 / 2} \leftrightarrow a^{6} S_{5 / 2}$ transition, unlike those to the $y^{6} P$ and $z^{6} P$ states, involves a two-electron transition from the ground $3 d^{5} 4 s^{2}$ configuration, and as such would be expected to exhibit a large matrix shift.

Vala and co-workers ${ }^{19}$ assigned features present at 330.0 and $345.7 \mathrm{~nm}$, under high metal atom loading to $\mathrm{Mn}$ dimer transitions from reversible temperature dependence effects observed in the recorded absorption spectra. These features were identified as $\mathrm{Mn}_{y}$ by Ozin and co-workers. ${ }^{21}$ The $\mathrm{Mn} / \mathrm{Ar}$ concentration study reported here, allows the assignment of the features at 226.4, 254.4, 311.6, and $402.3 \mathrm{~nm}$ to transitions of the manganese dimer.

\section{C. $\mathrm{Mn} / \mathrm{RG}$}

In this section the UV/Vis absorption spectra recorded for the most dilute $\mathrm{Mn} / \mathrm{RG}(\mathrm{RG}=\mathrm{Ar}, \mathrm{Kr}$, and $\mathrm{Xe}$ ) samples are presented. A high degree of atomic manganese isolation was achieved with the electron bombardment "metal sputtering" technique, and the absorption spectra presented in this

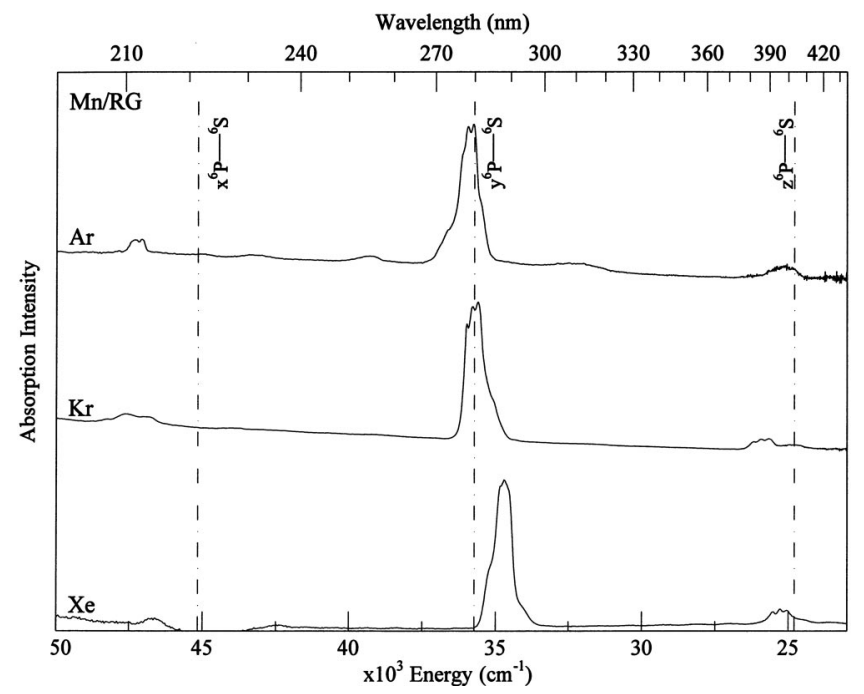

FIG. 2. UV/Vis absorption spectra recorded at $12 \mathrm{~K}$ following $\mathrm{Mn} / \mathrm{RG}$ sample deposition at $12 \mathrm{~K}$. The spectra shown correspond to the most dilute $\mathrm{Mn} / \mathrm{RG}$ samples prepared. The gas phase positions of the $x^{6} P_{5 / 2}, y^{6} P_{5 / 2}$, and $z^{6} P_{5 / 2} \leftarrow{ }^{6} S_{5 / 2}$ transitions are shown by the dashed vertical lines.

study are the most atomic reported to date. Trends evident in the spectra are highlighted in order to extract general conclusions on the site occupancy of atomic manganese in the solid rare gases. The vertical lines in Fig. 2 indicate the positions of the gas phase $x^{6} P_{5 / 2}, y^{6} P_{5 / 2}$, and $z^{6} P_{5 / 2} \leftrightarrow a^{6} S_{5 / 2}$ transitions of atomic Mn. The simplicity of the spectra and the location of the bands allow for easy assignment to the $y^{6} P_{5 / 2} \leftarrow a^{6} S_{5 / 2}$ and $z^{6} P_{5 / 2} \leftarrow a{ }^{6} S_{5 / 2}$ absorptions in RG matrices. As noted already in the $\mathrm{Mn} / \mathrm{Ar}$ system, assignment of the $x^{6} P_{5 / 2} \leftarrow a^{6} S_{5 / 2}$ transition is not as direct due to the large blue shift present on this 2-electron transition in the matrix. However, as shown in Fig. 2, it is observed in the $210 \mathrm{~nm}$ region for all three $\mathrm{Mn} / \mathrm{RG}$ systems investigated.

Line shape details of the absorption bands recorded for the $y^{6} P_{5 / 2} \leftarrow a{ }^{6} S_{5 / 2}$ and $z^{6} P_{5 / 2} \leftarrow a{ }^{6} S_{5 / 2}$ transitions of atomic $\mathrm{Mn}$ isolated in annealed RG solids are shown in Fig. 3. Inspection of the $y^{6} P_{5 / 2}$ state absorption, shown on the
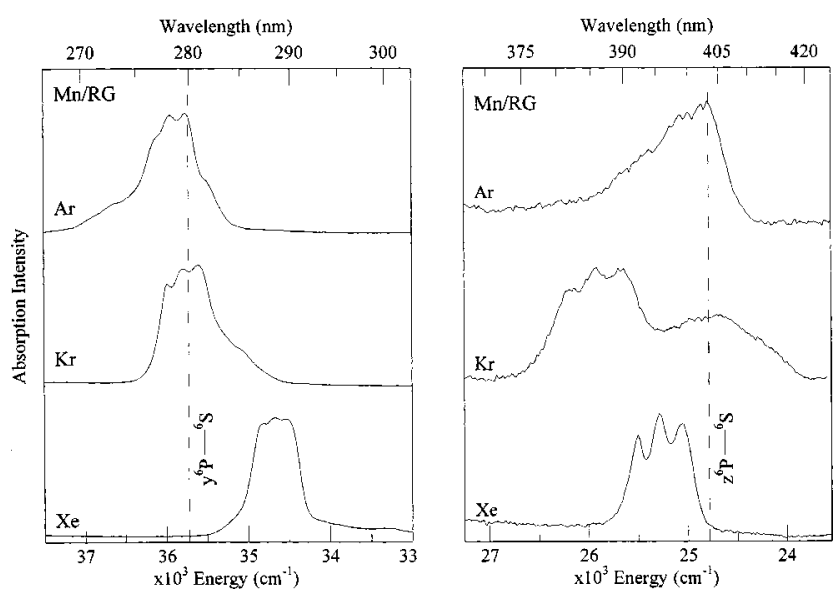

FIG. 3. Details of the UV and visible absorption spectra recorded following $\mathrm{Mn} / \mathrm{RG}$ sample annealing. The spectra shown correspond to the most dilute $\mathrm{Mn} / \mathrm{RG}$ samples prepared. The gas phase positions of the $y^{6} P_{5 / 2} \leftarrow{ }^{6} S_{5 / 2}$ and $z^{6} P_{5 / 2} \leftarrow{ }^{6} S_{5 / 2}$ transitions are indicated in the two panels. 
left of this figure, reveals a redshift of the band maximum from Ar to Xe but in an irregular manner. Thus the redshift calculated from the band maxima of $\mathrm{Ar}$ and $\mathrm{Kr}$ is only 151 $\mathrm{cm}^{-1}$, while that from $\mathrm{Kr}$ to $\mathrm{Xe}$ is $1109 \mathrm{~cm}^{-1}$. This irregular behavior can be rationalized in terms of the dominant/minor sites of $\mathrm{Mn}$ atom isolation in the different rare gas hosts. The dominant $\left(1^{\circ}\right)$ absorption feature in $\mathrm{Mn} / \mathrm{Kr}$ at $278 \mathrm{~nm}$ overlaps the gas phase transition and shows a weaker, red shoulder at $284.9 \mathrm{~nm}$. In solid Ar, the dominant $y^{6} P_{5 / 2} \leftarrow a{ }^{6} S_{5 / 2}$ absorption feature is at $278.1 \mathrm{~nm}$, showing a weaker, blue shoulder at $273.0 \mathrm{~nm}$. The latter band has been assigned by Vala and co-workers ${ }^{19}$ to a secondary site of isolation of $\mathrm{Mn}$ in argon. Comparison of the $\mathrm{Mn} / \mathrm{Ar}$ and $\mathrm{Mn} / \mathrm{Kr}$ absorption features suggests a site reversal where the dominant, blue site in $\mathrm{Kr}$ at $278.1 \mathrm{~nm}$ corresponds to the minor, blue site at 273.1 $\mathrm{nm}$ in solid Ar. When the minor, blue site in Ar is selected for comparison with the band maxima in $\mathrm{Kr}$ and $\mathrm{Xe}$, more regular matrix shifts of 823 and $1109 \mathrm{~cm}^{-1}$ are obtained.

The absorption spectra recorded for atomic manganese isolated in solid Xe after annealing to $35 \mathrm{~K}$ are, as shown in the lower trace of Fig. 3, different from $\mathrm{Ar}$ and $\mathrm{Kr}$ spectra in the following respects. First, only a single absorption band is present on the two transitions- $\mathrm{Ar}$ and $\mathrm{Kr}$ both exhibit pairs of bands. Second, the absorption feature centered at 288.2 $\mathrm{nm}$, assigned to the $y^{6} P_{5 / 2} \leftarrow a^{6} S_{5 / 2}$ transition, is redshifted in solid Xe from the gas phase position by $1028 \mathrm{~cm}^{-1}$. The two other hosts exhibited blueshifts on this transition. The threefold split band centred at $395.5 \mathrm{~nm}$, assigned to the $z^{6} P_{5 / 2} \leftarrow a^{6} S_{5 / 2}$ transition, exhibits a blueshift of $496 \mathrm{~cm}^{-1}$ from that of the free atom, behavior similar to that exhibited by $\mathrm{Ar}$ and $\mathrm{Kr}$ data for this transition.

Inspection of the visible absorptions recorded in the vicinity of the $z^{6} P_{5 / 2} \leftarrow a{ }^{6} S_{5 / 2}$ transition, shown on the right in Fig. 3, reveals behavior considerably more complex than that observed for the $y^{6} P_{5 / 2} \leftarrow a^{6} S_{5 / 2}$ transition. The absorption spectra indicate that a progressive redshift from $\mathrm{Ar}$ to $\mathrm{Xe}$ is not observed, as the $\mathrm{Mn} / \mathrm{Kr} z{ }^{6} P_{5 / 2} \leftarrow a^{6} S_{5 / 2}$ absorption appears to higher energy than the $\mathrm{Mn} / \mathrm{Ar}$ band. Extension of the site occupancy argument to the $z^{6} P_{5 / 2} \leftrightarrow a^{6} S_{5 / 2}$ transition predicts the existence of a secondary site of isolation in solid Ar to higher energy than the observed band maximum. However, this is not evident from the spectra shown on the right in Fig. 3. Identification of a secondary site, blueshifted from the dominant absorption in Ar, would rationalize the trends exhibited by the matrix shifts for the $z^{6} P_{5 / 2} \leftarrow a^{6} S_{5 / 2}$ absorption. However, the existence of a secondary high-energy site of atomic Mn isolation in solid Ar was only detected with $z^{6} P$ state excitation spectroscopy, the results of which are now presented.

\section{D. $z^{6} P$ state excitation spectroscopy}

High resolution excitation spectra recorded by monitoring the atomic emission features produced with steady-state excitation of the $z^{6} P_{5 / 2} \leftarrow a^{6} S_{5 / 2}$ transition for the three $\mathrm{Mn} / \mathrm{RG}$ systems are presented in Fig. 4. The photophysical characteristics of the monitored emission bands ${ }^{22}$ are collected in Table I as well as tentative state assignments. The excitation spectra were recorded in $\mathrm{Mn} / \mathrm{Ar}, \mathrm{Mn} / \mathrm{Kr}$, and

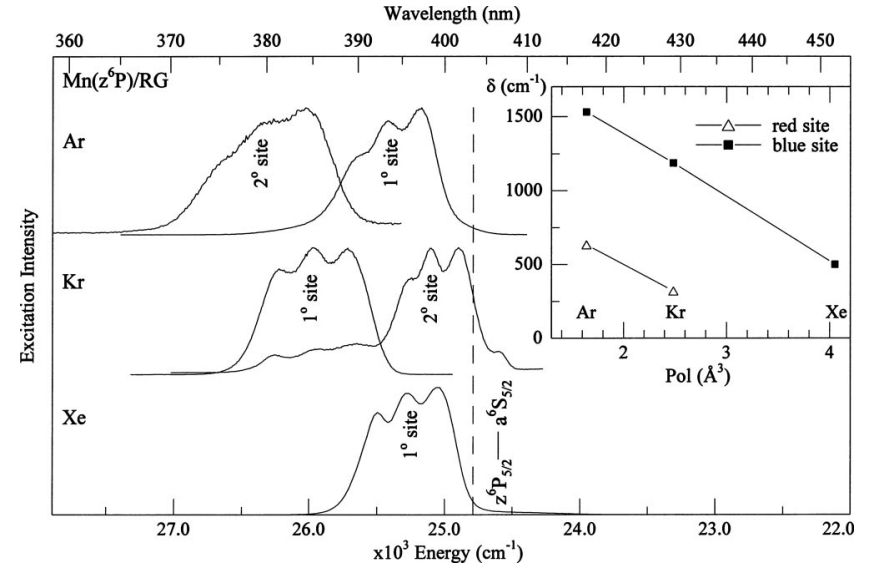

FIG. 4. Excitation spectra recorded in the vicinity of the $z^{6} P_{5 / 2} \leftarrow a{ }^{6} S_{5 / 2}$ transition for atomic manganese in $\mathrm{Ar}, \mathrm{Kr}$, and $\mathrm{Xe}$ after sample deposition at $12 \mathrm{~K}$ and matrix annealing. The excitation bands were recorded monitoring emission in $\mathrm{Mn} / \mathrm{Ar}$ at 413 and $428 \mathrm{~nm}$, in $\mathrm{Mn} / \mathrm{Kr}$ at 416 and $428 \mathrm{~nm}$ and in $\mathrm{Mn} / \mathrm{Xe}$ at $620 \mathrm{~nm}$. The inset shows a plot of the gas phase to Mn/RG matrix frequency shifts $\left(\delta \mathrm{cm}^{-1}\right)$ observed for the blue and red sites identified for the $z^{6} P_{5 / 2} \leftarrow a{ }^{6} S_{5 / 2}$ transition versus the RG host polarizabilities. The squares, connected by the solid line, highlight the linear correlation between the frequency shifts and rare gas polarizability observed for the $\mathrm{Mn} z{ }^{6} P_{5 / 2}$ $\leftarrow a{ }^{6} S_{5 / 2}$ transition occurring within the blue sites of isolation.

$\mathrm{Mn} / \mathrm{Xe}$ samples annealed to 29,42 , and $60 \mathrm{~K}$, respectively. Excitation scans of thermally unstable emission features (not shown) revealed broad bands, spanning the entire regions of the blue and red site features presented, but none extended to higher energy than the blue sites.

The excitation spectra shown in Fig. 4 reveal the presence of threefold split excitation patterns for each of the sites identified in all the RG solids. The observed splitting is attributed to the Jahn-Teller effect-indicative of Mn atom occupancy in highly symmetric ${ }^{24}$ matrix environments. Pairs of excitation bands are present in the $\mathrm{Mn} / \mathrm{Ar}$ and $\mathrm{Mn} / \mathrm{Kr}$ systems while only a single band is present in $\mathrm{Mn} / \mathrm{Xe}$. The improvement in the excitation spectra over the absorption spectra presented in Fig. 3, is most striking in the $\mathrm{Mn} / \mathrm{Ar}$

TABLE I. Photophysical characteristics of the matrix $\mathrm{Mn} / \mathrm{RG}$ emission bands monitored in the recording of the excitation spectra shown in Figs. 4-6. All the emission lifetimes quoted are the observed values with the exception of the nanosecond values, which are the radiative lifetimes corrected for the effective field of the solids. Tentative state assignments are also given, the details of which are presented in Ref. 22. The abbreviations $m$ and $s$ in column 3 denote the occurrence of multiple and single emission bands, respectively.

\begin{tabular}{lccccc}
\hline \hline & $\lambda_{\mathrm{em}}(\mathrm{nm})$ & No. bands & $\Delta_{\mathrm{em}}\left(\mathrm{cm}^{-1}\right)$ & $\tau_{\mathrm{em}}(\mathrm{ms})$ & State assign $^{\mathrm{a}}$ \\
\hline $\mathrm{Mn} / \mathrm{Ar}$ & 413 & $\mathrm{~s}$ & 465 & $57 \times 10^{-6}$ & $z{ }^{6} P$ \\
& 428 & $\mathrm{~m}$ & 7 & 25.3 & $a{ }^{4} D$ \\
& 590 & $\mathrm{~s}$ & 100 & 0.65 & $a{ }^{6} D$ \\
& 625 & $\mathrm{~s}$ & 260 & 0.17 & $a{ }^{6} D$ \\
$\mathrm{Mn} / \mathrm{Kr}$ & 416 & $\mathrm{~s}$ & 505 & $55 \times 10^{-6}$ & $z^{6} P$ \\
& 428 & $\mathrm{~m}$ & 6 & 23.9 & $a^{4} D$ \\
& 565 & $\mathrm{~s}$ & 523 & 0.112 & $z^{8} P$ \\
& 588 & $\mathrm{~m}$ & 65 & 0.29 & $a^{6} D$ \\
$\mathrm{Mn} / \mathrm{Xe}$ & 620 & $\mathrm{~s}$ & 240 & 1.83 & $a^{6} D$ \\
\hline \hline
\end{tabular}

${ }^{\text {aReference } 22 .}$ 
system. Thus a pair of distinct but overlapped excitation bands are obtained centered at 380 and $393 \mathrm{~nm}$, both of which exhibit threefold splitting. These features are present but not identifiable in the absorption spectra, shown on the right in Fig. 3, which has the added complication of a manganese dimer transition at $402 \mathrm{~nm}$. The dependence of the $z^{6} P_{5 / 2} \leftarrow a^{6} S_{5 / 2}$ transition energy on site occupancy is made evident in the excitation spectra shown in Fig. 4. Moreover, the progressive redshift from $\mathrm{Ar}$ to $\mathrm{Xe}$ is quite clear and the connection between the secondary (blue) site in $\mathrm{Mn} / \mathrm{Ar}$, the primary (blue) site in $\mathrm{Kr}$ and the single thermally stable site in $\mathrm{Mn} / \mathrm{Xe}$ is evident. A comparison of these spectra with the absorption spectra reveals the improvement in the site identification achievable with excitation spectroscopy.

To establish the connection between the blue sites in Ar and $\mathrm{Kr}$ and the single site in $\mathrm{Xe}$, a plot of the recorded matrix shifts vs the polarisabilities of the rare gases is presented in the inset of Fig. 4. The linear dependence exhibited in this plot for the three rare gas hosts reveals a correlation exists between the blue features and strongly suggests the same site occupancy of Mn atoms in the three hosts. Shown also in this plot is the matrix shift of the red sites in $\mathrm{Ar}$ and $\mathrm{Kr}$. A linear extrapolation of the red site data in $\mathrm{Ar}$ and $\mathrm{Kr}$, shown by the triangles in Fig. 4, clearly does not include the single site present in the Xe system. This indicates that the site producing the red bands in $\mathrm{Mn} / \mathrm{Ar}$ and $\mathrm{Mn} / \mathrm{Kr}$ is quite distinct from that occupied in $\mathrm{Mn} / \mathrm{Xe}$. Moreover, the much smaller matrixshift present on the red bands indicates a less repulsive interaction between the excited state $\mathrm{Mn}$ atom and its surroundings in this site than in the blue site. This suggests a larger site is responsible for the red absorption features than that giving rise to the blue absorptions.

\section{E. $y^{6} P$ state excitation spectroscopy}

High-resolution excitation spectra recorded in the vicinity of the $y^{6} P_{5 / 2} \leftarrow a{ }^{6} S_{5 / 2}$ transition monitoring site-specific atomic emission features in the $\mathrm{Mn} / \mathrm{RG}$ systems are shown in Fig. 5. Similar to the $z^{6} P_{5 / 2}$ state, all the UV excitation spectra recorded show resolved threefold split patterns indicative of $\mathrm{Mn}$ atom occupancy in high symmetry matrix sites for three $\mathrm{Mn} / \mathrm{RG}$ systems studied. Comparison of the $y^{6} P_{5 / 2}$ and $z^{6} P_{5 / 2}$ state excitation spectra, Figs. 5 and 4 , respectively, reveals the same site specificities. However, the nature of the matrix shifting is very different, with some of the $y^{6} P_{5 / 2}$ state bands now at lower energy than the gas phase transition. As observed in the absorption spectroscopy, the $y^{6} P_{5 / 2} \leftarrow a^{6} S_{5 / 2}$ transition occurring for Mn atoms isolated in the $1^{\circ}$ site of solid $\mathrm{Kr}$ overlaps the gas phase transition (indicated by the vertical line in Fig. 5), while for $\mathrm{Mn} / \mathrm{Ar}$ and $\mathrm{Mn} / \mathrm{Xe}$ the bands occur at higher and lower energies, respectively.

\section{F. $z^{8} P$ state excitation spectroscopy}

A summary of the site-specific excitation spectra recorded in the region of the gas phase $z^{8} P_{5 / 2}$ state by monitoring the red emission features of atomic Mn isolated in solid $\mathrm{Ar}, \mathrm{Kr}$, and $\mathrm{Xe}$ is presented in Fig. 6. The spectra shown were recorded with direct dye laser excitation of the

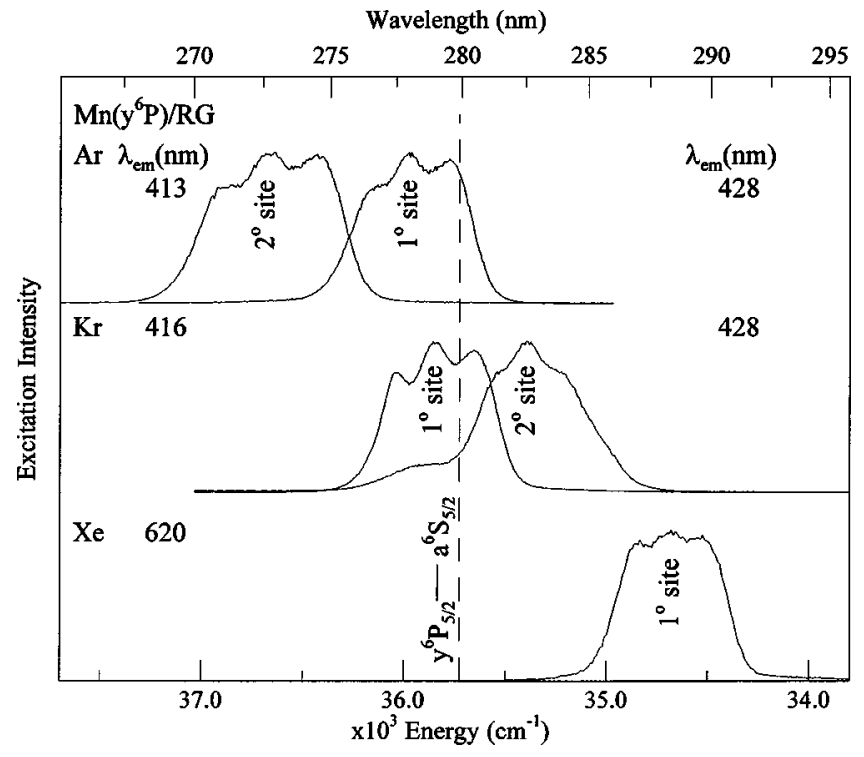

FIG. 5. Excitation spectra recorded for $\mathrm{Mn} / \mathrm{RG}$ samples in the vicinity of the $y^{6} P_{5 / 2} \leftrightarrow a^{6} S_{5 / 2}$ gas phase transition by monitoring the emission wavelengths indicated in the plot. $\mathrm{Mn} / \mathrm{RG}$ sample deposition was done at $12 \mathrm{~K}$ and spectra were recorded after matrix annealing.

spin forbidden $z^{8} P_{5 / 2} \leftarrow a{ }^{6} S_{5 / 2}$ transition. It is evident in Fig. 6 that all the excitation spectra exhibit bands with very well resolved threefold split patterns in all three rare gas matrices. The low intensity of the red components near $530 \mathrm{~nm}$ is a reflection of the weak output of the dye material (Coumarin 500) used in recording the spectra. Significantly all the $z^{8} P_{5 / 2} \leftarrow a{ }^{6} S_{5 / 2}$ transitions are blueshifted from the gas phase position and exhibit a progressive redshift from Ar to Xe by amounts similar to those observed for the $z^{6} P_{5 / 2}$ $\leftarrow a{ }^{6} S_{5 / 2}$ transition. This behavior is attributed to the "trip-

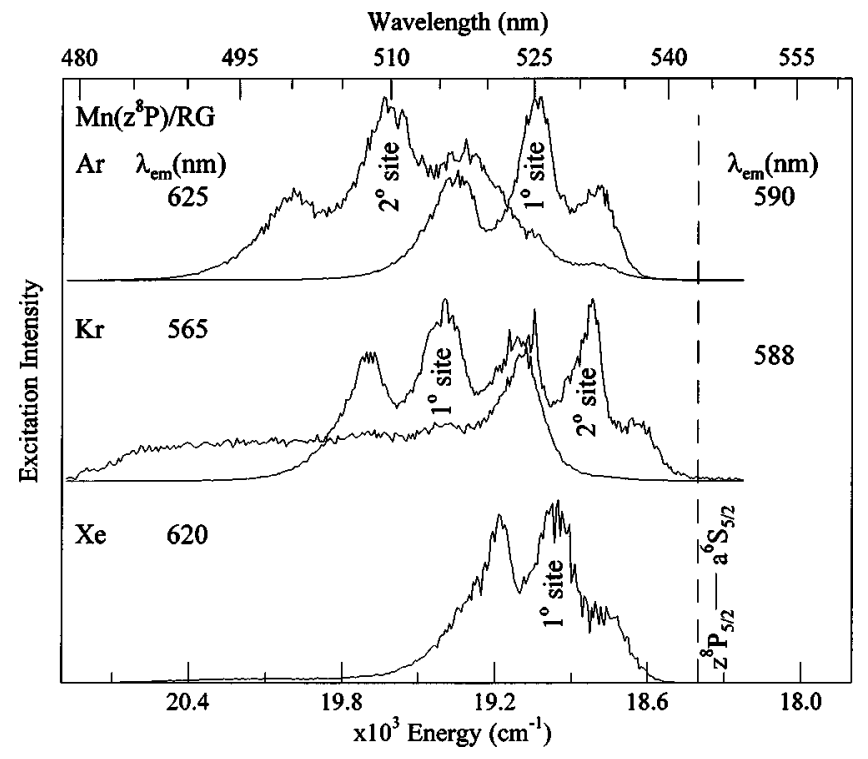

FIG. 6. $\mathrm{Mn} / \mathrm{RG}$ excitation spectra recorded at $12 \mathrm{~K}$ for all the $\mathrm{Mn} / \mathrm{RG}$ systems investigated produced with laser excitation in the vicinity of the $\operatorname{Mn} z{ }^{8} P_{5 / 2} \leftarrow a{ }^{6} S_{5 / 2}$ transition. The spectra shown were recorded monitoring emission bands as indicated by $\lambda_{\mathrm{em}}$ in wavelength units. The dashed vertical line indicates the position of the spin forbidden $z^{8} P_{5 / 2} \leftarrow a^{6} S_{5 / 2}$ gas phase transition of atomic $\mathrm{Mn}$ at $543.3 \mathrm{~nm}\left(18402 \mathrm{~cm}^{-1}\right)$. 


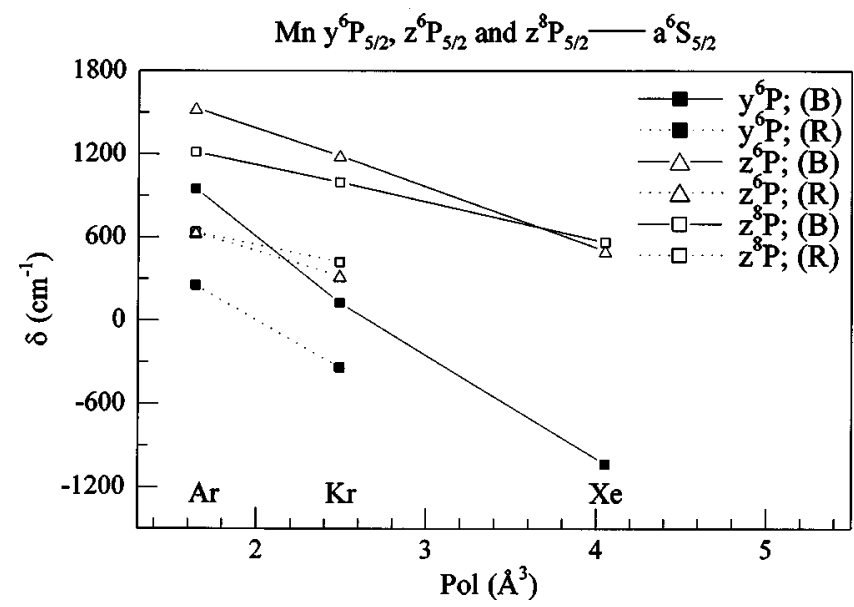

FIG. 7. A plot of the gas phase to Mn/RG matrix frequency shifts, $\delta\left(\mathrm{cm}^{-1}\right.$ units) observed for the blue and red sites identified for the three $P \leftarrow S$ transitions to the $y^{6} P, z^{6} P$, and $z^{8} P_{5 / 2}$ states of atomic manganese investigated in this study. The matrix shifts are plotted vs the RG host polarizability.

let" like nature of both the $z^{8} P$ and $z^{6} P$ excited states. A correlation of the blue sites in $\mathrm{Ar}$ and $\mathrm{Kr}$ and the single site in $\mathrm{Xe}$ is clearly evident.

\section{DISCUSSION}

\section{A. Matrix-shifts}

A comparison of the matrix shifts on the blue sites recorded for transitions to the $y^{6} P_{5 / 2}, z^{6} P_{5 / 2}$, and $z^{8} P_{5 / 2}$ excited states is made in Fig. 7. This is achieved by plotting the gas phase to matrix energy shifts for these three transitions, calculated from the central threefold split component for each of the thermally stable sites in the solid RG's (observed in the excitation spectroscopy and shown in Figs. 4-6), against host RG polarizability data given in Table II. From the linear behavior exhibited by the three $P \leftarrow S$ transitions studied, it is evident in Fig. 7, that a correlation exists between the high-energy sites present in $\mathrm{Ar}$ and $\mathrm{Kr}$ and the single site Xe. On the other hand, the red sites exhibit distinct behavior with smaller shifts and weaker dependence on the rare gas polarizability.

TABLE II. Site sizes (Ref. 31) in angstrom units (A) for the spherically symmetric single substitutional (ss) and tetravacancy ( $\left.T_{\text {vac }}\right)$ site types in the solid rare gases. The polarizabilities of the rare gases, $\alpha$, used in generating the plots of matrix shift vs polarisabilities are given in $\AA^{3}$ volume units. The ground state bond lengths of the known $\mathrm{M}\left(4 s^{2}{ }^{1} S_{0}\right) \cdot \mathrm{RG}$ diatomics $(\mathrm{M}=\mathrm{Ca}$ and $\mathrm{Zn})$ are also presented. The values for the $\operatorname{Mg}\left(3 s^{2}{ }^{1} S_{0}\right) \cdot \mathrm{RG}$ diatomics are intermediate between the $\mathrm{Ca}$ and $\mathrm{Zn}$ extremes and are selected to obtain estimates of the unknown $\mathrm{Mn}\left(4 s^{2} 3 d^{5}{ }^{6} S_{5 / 2}\right) \cdot \mathrm{RG}$ bond lengths.

\begin{tabular}{lcccccc}
\hline \hline $\mathrm{RG}$ & ss $(\AA)$ & $T_{\mathrm{Vac}}(\AA)$ & $\alpha\left(\AA^{3}\right)^{\mathrm{a}}$ & $\begin{array}{c}\mathrm{Ca} \cdot \mathrm{RG}, \\
r_{e}(\AA)^{\mathrm{b}}\end{array}$ & $\begin{array}{c}\mathrm{Mg} \cdot \mathrm{RG}, \\
r_{e}(\AA)^{\mathrm{c}}\end{array}$ & $\begin{array}{c}\mathrm{Zn} \cdot \mathrm{RG}, \\
r_{e}(\AA)^{\mathrm{c}}\end{array}$ \\
\hline $\mathrm{Ar}$ & 3.756 & 4.404 & 1.6411 & 4.86 & 4.49 & 4.18 \\
$\mathrm{Kr}$ & 3.991 & 4.679 & 2.4844 & $\cdots$ & $\cdots$ & 4.2 \\
$\mathrm{Xe}$ & 4.335 & 5.083 & 4.0440 & $\cdots$ & 4.56 & 4.4 \\
\hline \hline
\end{tabular}

${ }^{\mathrm{a}}$ Reference 27.

${ }^{\mathrm{b}}$ Reference 32.

${ }^{\mathrm{c}}$ Reference 6.

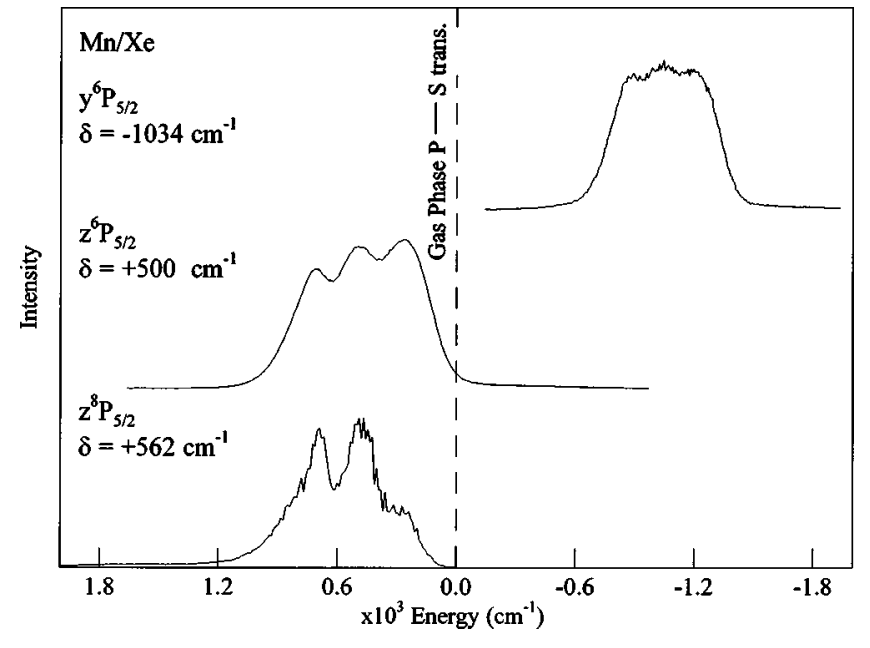

FIG. 8. Excitation spectra recorded for the $y^{6} P, z^{6} P$, and $z^{8} P_{5 / 2}$ states of $\mathrm{Mn}$ in Xe showing the matrix shifting relative to the gas phase position. The spectra were recorded by monitoring the atomic $620 \mathrm{~nm}$ emission feature in solid $\mathrm{Mn} / \mathrm{Xe}$. Note the excitation spectra are shown relative to the appropriate gas phase transition where the zero position represents the gas phase transitions involved.

Examination of the spectral positions recorded for the matrix excitation bands, corresponding to the $z^{6} P_{5 / 2}$ $\leftarrow a^{6} S_{5 / 2}$ and $y^{6} P_{5 / 2} \leftarrow a^{6} S_{5 / 2}$ transitions of atomic Mn, shown in Fig. 4 and Fig. 5, respectively, reveals that the former transition occurs to higher energy than the gas phase position in all cases. In contrast, the $y^{6} P_{5 / 2} \leftarrow a{ }^{6} S_{5 / 2}$ excitation features overlap the gas phase position for $\mathrm{Mn} / \mathrm{Kr}$, while the $\mathrm{Mn} / \mathrm{Ar}$ and $\mathrm{Mn} / \mathrm{Xe}$ bands occur at higher and lower energies respectively. As illustrated in Fig. 8, the differences in the matrix shifts observed for the three $P \leftarrow S$ type transitions are most pronounced in solid $\mathrm{Xe}$. In this figure, for which the three excitation spectra are zeroed with respect to the positions of their respective gas phase transitions, it is very evident that the $y^{6} P$ transition is redshifted while both the $z^{6} P$ and $z^{8} P$ transitions exhibit blue matrix shifts which are very similar in magnitude.

As the ground $a^{6} S_{5 / 2}$ state is common to all these excitation transitions, the contrasting behavior must originate from differences in the $y^{6} P$ and $z^{6} P / z^{8} P$ excited states. From this it can be concluded that the overall interaction of the excited state atom with the matrix environment must be more repulsive for the $z^{6} \mathrm{P} / z^{8} \mathrm{P}$ excited states than for the $y^{6} P_{5 / 2}$ state. This observation is attributed to the spin "singlet" characteristic of the $\left[3 d^{5} 4 s 4 p\left({ }^{1} P^{\circ}\right)\right] y^{6} P$ state and "triplet" characteristic of both the $\left[3 d^{5} 4 s 4 p\left({ }^{3} P^{\circ}\right)\right] z^{6} P$ and $\left[3 d^{5} 4 s 4 p\left({ }^{3} P^{\circ}\right)\right] z^{8} P$ states. From spectroscopic studies of the $n s^{2}$ metal atom/rare gas atom diatomic van der Waals molecules, it is known ${ }^{6}$ that the excited ${ }^{1} \Pi$ molecular state derived from the atomic spin singlet state exhibits a shorter bond length and a larger binding energy than its ${ }^{3} \Pi$ state equivalent. Thus the "singletlike" ${ }^{6} P_{5 / 2}$ state has a larger excited state stabilization thereby lowering the observed "singletlike" $y^{6} P_{5 / 2}$ transition energy and inducing a red matrix shift in Xe.

The contrasting behavior of the $y^{6} P_{5 / 2}$ and $z^{6} P / z^{8} P$ excited states is attributed to the dominance of the attractive ${ }^{1} \Pi$ like interaction over the repulsive ${ }^{1} \Sigma$ like occurring in 
the Franck-Condon (FC) accessible region of the $y^{6} P$ excited state. In the $z^{6} P$ and $z^{8} P$ excited states, the repulsive ${ }^{3} \Sigma$ like interaction dominates the weaker attraction of the ${ }^{3} \Pi$ state, resulting in the observed blue matrix shift. It is also evident in Fig. 8, that the difference in the matrix shifts observed in Xe matrices for both the $z^{6} P$ and $z^{8} P \leftarrow a{ }^{6} S$ transitions is only $62 \mathrm{~cm}^{-1}$. This effect is also manifested in Fig. 7 where the slopes of the polarizability plots of these two states are in close agreement and quite distinct from the $y^{6} \mathrm{P}$ excited state. This indicates that the interactions occurring in the Franck-Condon region of both $z^{6} P$ and $z^{8} P$ states are similar and determined by the spin triplet arrangement of the two electrons in the $4 s$ and $4 p$ orbitals. The different behavior in the $y^{6} P$ state can be traced back to the spin singlet arrangement of the $4 s 4 p$ orbital electrons.

\section{B. Site occupancy}

Because of its spatial symmetry, the $a^{6} S$ ground state of atomic manganese will favor isolation in spherical sites of isolation. Therefore, spherically symmetric trapping sites within the RG fcc lattice are considered the most likely candidates for atomic $\mathrm{Mn}$ isolation. This approach is supported by the characteristic threefold splitting observed in the bands present in all the $\mathrm{Mn} / \mathrm{RG}$ excitation spectra. This splitting has been established, from earlier MCD work, ${ }^{24}$ to arise from dynamic Jahn-Teller coupling between the excited $P$ state atom and the lattice when the guest atom resides in sites of high (cubic) symmetry. Linear (divacancy) and planar trivacancy sites have reduced dimensionality, so that even when relaxed, nonsymmetric splittings will arise on the excitation bands. Molecular dynamics simulations ${ }^{25}$ conducted on matrix-isolated atomic sodium reveal that in absorption (or excitation) these alternative site occupancies produce a pair of bands and a single band. Such features have been recorded in molecular beam work where barium atoms "picked-up" on the surface of large rare gas clusters ${ }^{26}$ exhibit such a $2+1$ structure. These band shapes have not been observed in any of the $\mathrm{Mn} / \mathrm{RG}$ excitation spectra recorded in this study. For these reasons, only sites larger in three dimensions such as expanded single substitutional and tetra-vacancy sites are considered for Mn atom occupancy. The simplest matrix system with respect to site occupancy is $\mathrm{Mn} / \mathrm{Xe}$, as only a single thermally stable site is present in the absorption and excitation spectra recorded for annealed samples. Xe also provides the best starting point for a site analysis, because as indicated by the data collected in Table II, it represents the ideal matrix host for atomic isolation due to the larger sites it has available.

A significant difficulty encountered in an attempt to identify the site occupancy of atomic manganese in the solid rare gases is the lack of any information, either theoretical or experimental, on the diatomic $\mathrm{Mn} \cdot \mathrm{RG}$ ground state bond lengths. In the absence of this data, we will utilize the information that exists on the $\mathrm{Mg} \cdot \mathrm{RG}$ diatomics. The $\mathrm{Mg} \cdot \mathrm{RG}$ systems are chosen on the basis of the similar dispersion (London) forces predicted in the Mn $\cdot \mathrm{RG}$ diatomics because of the similar polarisabilities and ionization potentials of these two metal atoms. Thus $\mathrm{Mg}$ and Mn have $\alpha$ values $^{27}$ of 10.6 and $9.4 \AA^{3}$, respectively while their IP's are 61671 and

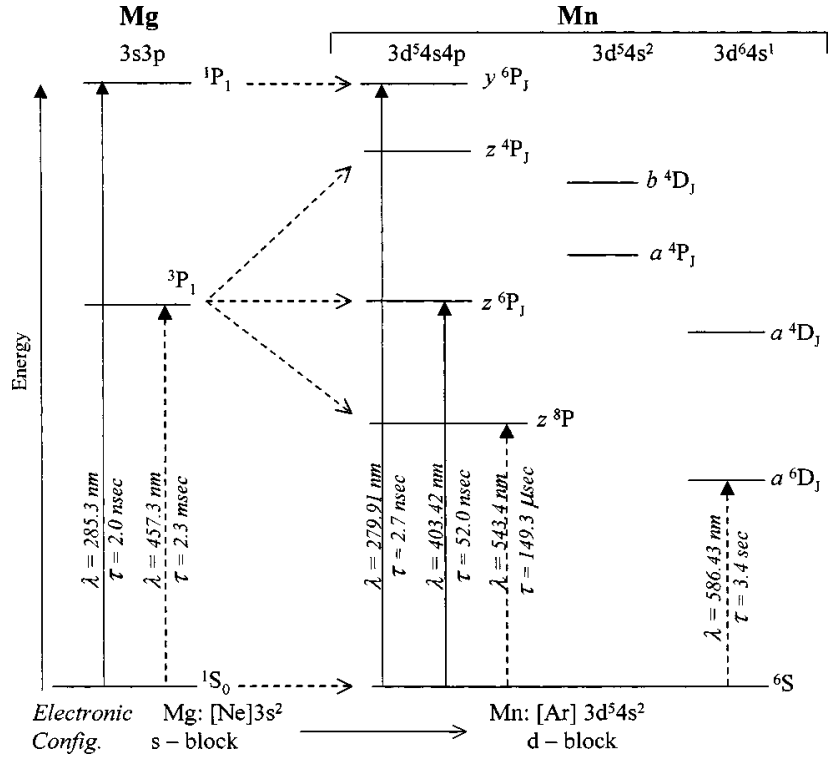

FIG. 9. An energy level diagram showing the correspondence between the $z^{6} P_{J} /{ }^{3} P_{J}$ levels of atomic magnesium and the ${ }^{6} P_{J} /{ }^{1} P_{1}$ levels of atomic manganese. The allowed $y^{6} P_{5 / 2} \leftarrow a^{6} S_{5 / 2}$ and $z^{6} P_{5 / 2} \leftarrow a^{6} S_{5 / 2}$ transitions of Mn occur at $35726 \mathrm{~cm}^{-1}(279.91 \mathrm{~nm})$ and $24788 \mathrm{~cm}^{-1}(403.42 \mathrm{~nm})$, respectively, and are indicated by solid arrows. The allowed ${ }^{1} P_{1} \leftarrow{ }^{1} S_{0}$ and spin forbidden ${ }^{3} P_{1} \leftarrow{ }^{1} S_{0}$ transitions of Mg occur at $35051 \mathrm{~cm}^{-1}(285.3 \mathrm{~nm})$ and $21870 \mathrm{~cm}^{-1}(457.3 \mathrm{~nm})$ and are indicated by solid and broken arrows, respectively. Other states of $\mathrm{Mn}$ arising from rearrangement of the $d$-orbital electrons are shown on the right for completeness.

$59959 \mathrm{~cm}^{-1}$. Strong similarities also exist between the energetics of the ${ }^{1} P$ and ${ }^{3} P$ states of atomic $\mathrm{Mg}$ and the singletlike $y^{6} P$ and tripletlike $z^{6} P$ states of atomic Mn. Thus, as shown in Fig. 9, the gas phase ${ }^{1} P$ and ${ }^{3} P \leftrightarrow{ }^{1} S$ transitions of atomic $\mathrm{Mg}$ occur at 285.3 and $457.3 \mathrm{~nm}$, respectively, while the corresponding $y^{6} \mathrm{P}$ and $z^{6} \mathrm{P} \leftrightarrow a^{6} S$ transitions of $\mathrm{Mn}$ occur at 279.9 and $403.4 \mathrm{~nm}$.

Contrary to what might be expected on the basis of the numbers of valence electrons, the spectroscopically determined ground state bond lengths of the Group 2 (IIA) $\mathrm{M} \cdot \mathrm{RG}$ diatomics are consistently larger than their Group 12 (IIB) counterparts. As indicated in Table II, $\mathrm{Mg} \cdot \mathrm{Ar}$ and $\mathrm{Mg} \cdot \mathrm{Xe}$ have bond lengths of 4.49 and $4.56 \AA$, respectively, while the corresponding $\mathrm{Zn} \cdot \mathrm{Ar}$ and $\mathrm{Zn} \cdot \mathrm{Xe}$ values are 4.18 and $4.44 \AA$, respectively. Rotationally resolved data does not exist yet on the $\mathrm{Ca} \cdot \mathrm{RG}$ diatomics, however, high level $a b$ initio calculations on ground state $\mathrm{Ca} \cdot \mathrm{Ar}$ consistently show a long bond length of $4.86 \AA$. Consideration of the values supports the selection of $\mathrm{Mg}$ as the best guide for the still unavailable $\mathrm{Mn} \cdot \mathrm{RG}$ ground state bond lengths.

The Mg atom exhibits a $3 s^{2}$ ground state electronic configuration while that of $\mathrm{Mn}$ is $3 d^{5} 4 s^{2}$. The presence of the compact, half-filled $3 d^{5}$ subshell and the small difference in energy between the $4 s$ orbital of $\mathrm{Mn}$ and the $3 s$ orbital of $\mathrm{Mg}$ makes this comparison feasible. Accordingly, the $\mathrm{Mg} \cdot \mathrm{RG}$ diatomic ground state bond lengths, known for 1:1 complexes $(\mathrm{RG}=\mathrm{Ar}$ and $\mathrm{Xe})$ prepared in supersonic expansions, are expected to be similar to those of the $\mathrm{Mn} \cdot \mathrm{RG}$ systems.

The $\mathrm{Mg} \cdot \mathrm{RG}(\mathrm{RG}=\mathrm{Ar}$ and $\mathrm{Xe})$ ground state bond lengths $\left(r_{e}\right)$ are presented in Table II. Comparison of these bond 
lengths with the site sizes available in the solid rare gases reveals isolation of $\mathrm{Mg}$ atoms (and therefore $\mathrm{Mn}$ ) either in deformed substitutional sites or tetra-vacancy sites in solid $\mathrm{Xe}$. The presence of a single thermally stable site of isolation in solid Xe shows the preference for the Mn atoms for a particular site type. However, a definitive assignment is not possible based solely on application of the $\mathrm{Mg} \cdot \mathrm{Xe}$ ground state bond length to the Mn system, as the comparison reveals the possibility of tetravacancy site occupancy. Therefore, trends exhibited by $\mathrm{Mn}$ atoms isolated in solid $\mathrm{Ar}$ and $\mathrm{Kr}$ must be used to validate any assignment of site occupancy. In solid Ar, the red site dominates the $y{ }^{6} P$ state absorption spectra, while the dominant site of isolation in solid $\mathrm{Kr}$ is the blue site. This difference represents a reversal of the preferred site occupancy in $\mathrm{Mn} / \mathrm{Ar}$ and $\mathrm{Mn} / \mathrm{Kr}$. To identify the trends in the site occupancy and the relationship of thermally stable sites of isolation in each RG solid, conclusions drawn from the polarizability model ${ }^{23}$ are now employed.

When the polarizability model is applied to all the blue sites identified on the $z^{6} P \leftarrow a{ }^{6} S, \quad{ }^{6} P \leftarrow a{ }^{6} S$ and $z^{8} P$ $\leftarrow a^{6} S$ transitions, the results shown in Fig. 7 are obtained. It is evident from the linear relationship between the matrix shifts and the host polarizability, that the blue sites correspond to the same matrix-trapping environment in all three hosts. The red site, which dominates the Mn/Ar excitation spectra, is correlated with the red, but minor site in Kr. The site dominance is therefore reversed from red to blue from $\mathrm{Mn} / \mathrm{Ar}$ to $\mathrm{Mn} / \mathrm{Kr}$ and a correlation exists between the blue sites identified in all three rare gas hosts. The red/blue site dominance is attributed to the preference for a different site type in the heavier RG solids. A comparison of the $\mathrm{Mg} \cdot \mathrm{Ar}$ ground state bond length $(4.49 \AA)$ with the site size available for the tetra-vacancy in solid Ar (4.404 $\AA$ ) and the substitutional site $(3.756 \AA)$ reveals a favorable match with the former site but a large size mismatch with the latter. Therefore the red sites of atomic $\mathrm{Mn}$ isolation in solid $\mathrm{Ar}$ and $\mathrm{Kr}$ are assigned to $\mathrm{Mn}$ atom trapping in tetra-vacancy sites. The preference for a single site in solid $\mathrm{Xe}$, and the correlation of the matrix-shifts observed for the blue sites, allows the assignment of the blue sites to the trapping of $\mathrm{Mn}$ atoms in single substitutional sites of these matrices. Our previous simulation work on the matrix-isolated Group 12 metal atoms $\mathrm{Zn},{ }^{5} \mathrm{Cd},{ }^{28}$ and $\mathrm{Hg},{ }^{29}$ indicated single substitutional site occupancies for these $\mathrm{M} \cdot \mathrm{RG}$ diatomics that have comparatively short bond lengths. The present predictions for the site occupancies in the $\mathrm{Mn} / \mathrm{RG}$ matrix systems are consistent with the earlier simulations because of the longer ground state bond length expected in manganese-rare gas diatomics.

\section{Dependence of matrix-shift on spin state}

Laursen and Cartland's application of the polarizability model to the Group 12 metal atoms ${ }^{23} \mathrm{Zn}, \mathrm{Cd}$, and $\mathrm{Hg}$ undergoing ${ }^{1} P_{1} \leftarrow{ }^{1} S_{0}$ and ${ }^{3} P_{1} \leftarrow{ }^{1} S_{0}$ transitions revealed that the frequency shifts of the absorption bands were approximately linear with the RG polarizability. This has also been shown to be the case for the $P \leftarrow S$ type electronic transitions of atomic $\mathrm{Mn}$ and was achieved in the previous sections by the correlation of the blue sites in the three matrices and red sites in $\mathrm{Ar}$ and $\mathrm{Kr}$. However L\&C also observed that the slope of the linear relationship of the matrix shifts was dependent on the multiplicity of the excited state. This is also observed for $\mathrm{Mn} / \mathrm{RG}$ solids because, as shown in Fig. 7, the slope of the "singlet" like $y^{6} P$ state is much greater than the "triplet" like $z^{6} P$ and $z^{8} P$ states. This effect is attributed to the different $\Pi$ and $\Sigma$ excited state interactions occurring for the $\mathrm{Mn}$ atom in a particular site of isolation. The blue site for the $y^{6} P \leftarrow a{ }^{6} S$ state transition occurs to higher and lower energy than the gas phase transition from Ar to Xe respectively, whereas the $z^{6} P \leftarrow a^{6} S$ transition occurs to higher energy than the gas phase in all three hosts. The "triplet" like nature of the $z^{8} P \leftarrow a^{6} S$ transition is also evident in Fig. 7 as the transition energy occurs to higher energy than the gas phase in all $\mathrm{Mn} / \mathrm{RG}$ systems.

\section{CONCLUSIONS}

The UV/V is absorption spectroscopy reported for atomic manganese isolated in solid $\mathrm{Ar}, \mathrm{Kr}$, and $\mathrm{Xe}$ allowed the assignment of the $x, y$, and $z^{6} P_{5 / 2} \leftarrow a^{6} S_{5 / 2}$ transitions in each of the RG hosts. Pairs of thermally stable sites of isolation were identified for $\mathrm{Mn}$ atoms in solid $\mathrm{Ar}$ and $\mathrm{Kr}$ whereas single site occupancy was found in solid Xe. Additional absorption features present in samples with higher metal loadings are assigned to $\mathrm{Mn}_{2}$ transitions only. The spectra recorded for the $\mathrm{Mn} / \mathrm{RG}$ samples prepared in this study by electron bombardment show no evidence for the production of Mn aggregates larger than dimer.

The UV absorption features recorded for $\mathrm{Mn} / \mathrm{RG}$ solids in the vicinity of the $y^{6} P_{5 / 2} \leftrightarrow a^{6} S_{5 / 2}$ gas phase transition provided more direct information on the atomic trapping sites than the corresponding $z^{6} P_{5 / 2} \leftrightarrow a^{6} S_{5 / 2}$ transition. This is in part due to the increased oscillator strength ${ }^{30}$ of the "singlet" like $y^{6} P_{5 / 2} \leftarrow a{ }^{6} S_{5 / 2}$ transition over the "triplet" like $z^{6} P_{5 / 2}$ transition but is also due to the spectral congestion on the latter transition due to existence of a Mn dimer absorption at $402 \mathrm{~nm}$. The absorption bands assigned to the $y^{6} P_{5 / 2}$ state exhibited a redshift of the band maximum from Ar to $\mathrm{Kr}$ to $\mathrm{Xe}$ which deviate from linearity, consistent with the reversal of red dominant/blue minor sites of isolation from Ar to Xe observed on the $z^{6} P_{5 / 2} \leftarrow a{ }^{6} S_{5 / 2}$ transition. In solid Ar, the two thermally stable $y^{6} P_{5 / 2} \leftarrow a^{6} S_{5 / 2}$ absorption features were identified occurring to higher energy than the gas phase transition at 273 and $278.1 \mathrm{~nm}$. In $\mathrm{Kr}$ the $1^{\circ} \mathrm{Mn} / \mathrm{Kr}$ absorption feature at $279.9 \mathrm{~nm}$ overlaps the gas phase transition and the $2^{\circ}$ site occurred to lower energy. In solid Xe high temperature deposition and matrix annealing experiments allowed the definitive identification of the band located at $288.2 \mathrm{~nm}$ to a single site.

Excitation spectra provided clear-cut results for the existence of distinct site occupancies of atomic Mn in RG solids. Matrix shift data collected for the $y^{6} P, z^{6} P$, and $z^{8} P$ states allowed the association of these sites when polarizability plots of the matrix shifts on the $P \leftarrow S$ type transitions were made. The correlation of the high-energy blue sites in $\mathrm{Ar}$ and $\mathrm{Kr}$ with the single site in solid $\mathrm{Xe}$ was established and subsequent assignment of this site to $\mathrm{Mn}$ atoms in single substitutional sites was achieved. The analysis also allowed the grouping of the low energy red sites in $\mathrm{Ar}$ and $\mathrm{Kr}$ and their assignment to $\mathrm{Mn}$ atoms isolated in matrix tetra- 
vacancies. This was achieved using a comparison of the $\mathrm{Mg} \cdot \mathrm{RG}$ ground state bond lengths assuming the transference of the known $\mathrm{Mg} \cdot \mathrm{RG}$ parameters to the $\mathrm{Mn} \cdot \mathrm{RG}$ systems. The recorded absorption intensities showed that $\mathrm{Mn}$ atoms in solid Ar exhibit a preference for trapping in tetra-vacancy sites whereas single substitutional site occupancy is preferred in $\mathrm{Kr}$, and this site is the only thermally stable site in solid Xe.

The application of the polarizability model to the $y^{6} P$ $\leftarrow a{ }^{6} S, z^{6} P \leftarrow a{ }^{6} S$, and $z^{8} P \leftarrow a{ }^{6} S$ transitions of atomic Mn showed the same overall trends as the Group $12 n s^{2}$ metal atoms investigated by Laursen and Cartland ${ }^{23}$ insofar as the behavior evident on the $y^{6} P \leftarrow a{ }^{6} S$ and $z{ }^{6} P \leftarrow a{ }^{6} S$ matrix shifts mirrored those reported for the ${ }^{1} P \leftarrow{ }^{1} S$ and ${ }^{3} \mathrm{P} \leftarrow{ }^{1} S$ transitions of $\mathrm{Zn}, \mathrm{Cd}$, and $\mathrm{Hg} .{ }^{23}$ This highlighted the differences in the relative contributions from the $\Pi$ and $\Sigma$ excited states in the Franck-Condon accessible regions to the observed matrix shifts. The importance of the excited state multiplicity on the observed matrix shifts was evident in comparison of polarizability plots for the "singlet" like $y^{6} P \longleftarrow a^{6} S$ and "triplet" $z{ }^{6} P \leftarrow a^{6} S$ and $z^{8} P \leftarrow a^{6} S$ transitions. In addition the analysis of the of $z^{6} P \leftarrow a^{6} S$ and $z^{8} P \leftarrow a^{6} S$ transition energies reflected the similarity of the excited state interactions for these states.

\section{ACKNOWLEDGMENTS}

This research was funded by the Irish Government Enterprise Ireland, SC/98/403 Basic Science research grant to whom MC gratefully acknowledges receipt of a Ph.D. studentship.

${ }^{1}$ J. G. McCaffrey and G. A. Ozin, J. Chem. Phys. 101, 10354 (1994).

${ }^{2}$ V. A. Braken, P. Gürtler, and J. G. McCaffrey, J. Chem. Phys. 107, 5290 (1997).

${ }^{3}$ B. Healy and J. G. McCaffrey, J. Chem. Phys. 110, 3903 (1999).

${ }^{4}$ M. A. Collier and J. G. McCaffrey, J. Chem. Phys. 119, 11878 (2003).

${ }^{5}$ J. G. McCaffrey and P. N. Kerins, J. Chem. Phys. 106, 7885 (1997).

${ }^{6}$ C. Crepin and A. Tramer, J. Chem. Phys. 97, 4772 (1992).

${ }^{7}$ W. H. Breckenridge, C. Jouvet, and B. Soep, Advances in Metal and
Semiconductor Clusters, edited by M. A. Duncan (JAI, Greenwich, 1995), Vol. III.

${ }^{8}$ C. Crepin-Gilbert and A. Tramer, Int. Rev. Phys. Chem. 18, 485 (1999).

${ }^{9}$ G. Rojas-Lorenzo, J. Rubayo-Soneira, S. Fernandez-Alberti, and M. Chergui, J. Phys. Chem. A 107, 8225 (2003).

${ }^{10}$ N.I.S.T. Atomic Spectra Database, http://physics.nist.gov/cgi-bin/AtData/ (Website last accessed February 4, 2004).

${ }^{11}$ The quoted energies correspond to the transition from the ground $a{ }^{6} S_{5 / 2}$ state to the $J=\frac{5}{2}$ spin-orbit level of the $y^{6} P$ and $z^{6} P$ excited states.

${ }^{12}$ O. Schnepp, J. Phys. Chem. Solids 17, 188 (1960).

${ }^{13}$ M. McCarty and G. W. Robinson, Mol. Phys. 2, 415 (1959).

${ }^{14}$ E. L. Lee and R. G. Gutmacher, J. Phys. Chem. Solids 23, 1823 (1962).

${ }^{15}$ D. M. Mann and H. P. Broida, J. Chem. Phys. 55, 84 (1971).

${ }^{16}$ D. M. Gruen, "Spectroscopic Identification and Characterization of Matrix Isolated Atoms," in Cryochemistry, edited by M. Moskovitz and G. A. Ozin (Wiley-Interscience, New York, 1976), Chap. 10.

${ }^{17}$ J. Shakhsemampour, Ph.D. thesis, University of Florida, 1983 (unpublished).

${ }^{18}$ T. C. De Vore, A. Ewing, H. F. Franzen, and V. Calder, Chem. Phys. Lett. 35, 78 (1975).

${ }^{19}$ J. C. Rivoal, J. Shakhsemampour, K. K. Zeringue, and M. Vala, Chem. Phys. Lett. 92, 313 (1982).

${ }^{20}$ A. D. Kirkwood, K. D. Bier, J. K. Thompson, T. L. Haslett, A. S. Huber, and M. Moskovits, J. Phys. Chem. 95, 2644 (1991).

${ }^{21}$ W. E. Klotzbücher and G. A. Ozin, Inorg. Chem. 19, 3776 (1979).

${ }^{22}$ M. A. Collier and J. G. McCaffrey, J. Chem. Phys. (to be published); M. A. Collier, Ph.D. thesis, National University of Ireland, Maynooth, 2004

${ }^{23}$ S. L. Laursen and H. E. Cartland, J. Chem. Phys. 95, 4751 (1991).

${ }^{24}$ J. Rose, D. Smith, B. E. Williamson, P. N. Schatz, and M. C. M. O'Brien, J. Phys. Chem. 90, 2608 (1991).

${ }^{25}$ M. Ryan, P. DePujo, C. Crepin, and J. G. McCaffrey (unpublished results, 2004).

${ }^{26}$ J.-P. Visticot, P. DePujo, J.-M. Mestdagh, A. Lallement, J. Berlande, O. Sublemontier, P. Meynardier, and J. Curveillier, J. Chem. Phys. 100, 158 (1994).

${ }^{27}$ T. M. Miller, Handbook of Chemistry and Physics, 76th ed., edited by D. R. Lide (CRC, Boca Raton, 1995-1996).

${ }^{28}$ B. Healy and J. G. McCaffrey, J. Phys. Chem. A 104, 3553 (2000).

${ }^{29}$ M. A. Collier and J. G. McCaffrey, J. Chem. Phys. 119, 11888 (2003).

${ }^{30} \mathrm{In}$ solid $\mathrm{Xe}$, the ratio of the absorption intensity for the ${ }^{6}{ }^{6} P_{5 / 2}$ and $z^{6} P_{5 / 2} \leftarrow a{ }^{6} S_{5 / 2} I_{\mathrm{Abs}}\left(y^{6} P\right): I_{\mathrm{Abs}}\left(z^{6} P\right)$ was found to be $14: 1$ providing a measure of the relative oscillator strengths.

${ }^{31}$ H. E. Hallam, Vibrational Spectroscopy of Trapped Species (WileyInterscience, New York, 1973).

${ }^{32}$ F. Spiegelman, L. Maron, W. H. Breckenridge, J.-M. Mestdagh, and J.-P. Visticot, J. Chem. Phys. 117, 7534 (2002), and references cited therein. 